\title{
Abordagem comunitária e ensino médico: análise da experiência do DMIF-FCM-UERJ
}

\author{
Cesar A. O. Favoreto ${ }^{1 *}$
}

\section{Resumo}

Este ensaio analisa os aspectos conceituais e técnico-políticos da promoção da saúde e educação em saúde na aprendizagem médica da abordagem comunitária. Desenvolve-se a compreensão da abordagem comunitária como processo de construção da cidadania e de promoção da autonomia sobre o processo saúde-adoecimento. A partir desta reflexão, contextualiza e problematiza a experiência do Departamento de Medicina Integral e Familiar e Comunitária da Faculdade de Ciências Médicas da Universidade do Eestado do Rio de Janeiro (DMIF-FCM-UERJ) de deslocar o ensino médico para as unidades de atenção primária e comunidades adscritas. Na formação médica, a abordagem comunitária possibilita o desenvolvimento de uma nova e ampliada perspectiva semiológica, propedêutica e terapêutica da prática médica, fomenta para o aluno um espaço de aprendizagem que busca desenvolver a reflexão, a escuta e o diálogo com os profissionais e os pacientes e integra a dimensão individual e coletiva de promoção, prevenção e atenção à saúde.

Descritores: Educação médica; Promoção da saúde; Educação em saúde.

\section{Abstract \\ Community approach and medical education: analysis of the experience of the DMIF-FCM-UERJ}

This essay analyzes the conceptual, technical, and political aspects of health promotion and health education in medical learning under the community approach. It develops the understanding of the community approach as a process of building citizenship and the promotion of autonomy over the health-illness. From this reflection, it contextualizes and discusses the experience of the Department of Integrative Medicine and Family and Community of the School of Medical Sciences at UERJ (DMIF-FCM) moving the medical education towards primary care units and ascribed communities. In medical training, the community approach enables the development of a new and extended semiology, propaedeutic and therapeutic perspective of medical practice, provides the student a learning space that seeks to develop reflection, listening and dialogue with professionals, and patients, integrating the individual and collective dimension of promotion,
1. Departamento de Medicina Integral, Familiar e Comunitária. Faculdade de Ciências Médicas. Universidade do Estado do Rio de Janeiro. Rio de Janeiro, RJ, Brasil.

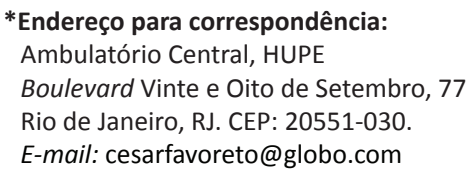

Revista HUPE, Rio de Janeiro, 2016;15(3):271-277 doi: $10.12957 /$ rhupe.2016.29472

Recebido em 27/06/2016. Aprovado em 04/11/2016.

prevention and health care.

Keywords: Medical education; Health promotion; Health education.

\section{Resumen}

Enfoque comunitario y la educación médica: análisis de la experiencia de la MIF-FCM-UERJ

Este ensayo analiza los aspectos conceptuales, técnica y política de la promoción y educación para la salud en el aprendizaje médico y en el enfoque de la comunidad. Se desarrolla una comprensión del enfoque comunitario como un proceso de construcción de la ciudadanía y la promoción de la autonomía sobre el proceso salud-enfermedad. A partir de esta reflexión, se contextualiza y analiza la experiencia del Departamento de Medicina Integral y Familiar y de la Comunidad de la Escuela de Ciencias Médicas de la UERJ (DMIF-FCM) para llevar la educación médica para las unidades de atención primaria y las comunidades adscritas. En la educación médica el enfoque comunitario permite el desarrollo de una nueva y amplia perspectiva semiótica, el proceso y la práctica de la terapia médica, fomenta en los estudiantes un espacio de aprendizaje que busca desarrollar la reflexión, la escucha y el diálogo con los profesionales y los pacientes, integra la promoción individual con la colectiva y la prevención con la atención de la salud.

Palabras clave: Educación médica; Promoción de la salud; Educación para la salud. 


\section{Relato exper}

\section{Abordagem comunitária e ensino médico: análise da experiência do DMIF-FCM-UERJ}

Este ensaio busca analisar os aspectos conceituais, técnico políticos e pedagógicos que orientaram a inserção da abordagem comunitária no ensino médico na experiência do Departamento de Medicina Integral, Familiar e Comunitário da Faculdade de Ciências Médicas da UERJ (DMIF-FCM). Analisa como estas questões teóricas se desdobraram em um processo de ensino-aprendizagem que envolve mudanças de contexto, de objetivos educacionais e de metodologias. Um processo que implica e repercute em ações de integração ensino-serviço que representam muito mais do que o deslocamento do ensino médico para as unidades de atenção primária e para as comunidades.

A necessidade da FCM de se adequar às diretrizes para o ensino médico, implantadas com as Diretrizes Curriculares Nacionais de 2001,1 induziu a inserção de conteúdos e competências da atenção primária no currículo da medicina por meio da criação, em 2002, das disciplinas de medicina integral nos primeiros semestres da graduação, entre elas, a disciplina de Abordagem Comunitária, Educação/Promoção à Saúde.

A escolha da disciplina de medicina integral (existente no internato desde 1976) para atender às orientações relativas ao ensino da Atenção Primária à Saúde (APS) decorreu do envolvimento e da experiência acadêmica de seu corpo docente. Estes professores defendiam há décadas mudanças curriculares como a desospitalização do ensino, a formação generalista e o compromisso da escola médica com a saúde pública.

A trajetória acadêmica destes docentes na APS estrutura-se desde 1976, com as experiências na residência de medicina integral, com o ensino da prática ambulatorial de medicina integral no ambulatório do Hospital Universitário Pedro Ernesto (atividade que permanece como campo de aprendizagem dos internos da FCM). Além disso, havia também ações de integração docente-assistencial envolvendo a residência e graduação em cenários extra-hospitalares como o Projeto Austin (localidade de Nova Iguaçu-RJ campo de atuação da residência de medicina integral e de preventiva e social no período de 1978 a 1983), o ambulatório comunitário do Morro do Borel (Tijuca, RJ) no período de 1992 a 1998 e o Centro Municipal de Saúde Bicuíba (no bairro de Lins de Vasconcelos, RJ), por último, o projeto Saúde na Vila, que territorializou e adscreveu moradores de Vila Isabel (RJ) para o acompanhamento dos residentes de medicina de família comunidade.

Estas experiências comunitárias fundamentaram a formação da disciplina de abordagem comunitária, denominada disciplina de Medicina Integral I, que é voltada para o diagnóstico da situação de saúde de uma comunidade e organização de ações de educação e a promoção da saúde. Além de envolver experiências de integração docente assistencial, esta disciplina se organizou com base em reflexões críticas ao modelo flexneriano de ensino médico que é a base paradigmática de uma visão descontextualizada e fragmentada do processo saúde-adoecimento.

Este foi um movimento que dialogou com as Diretrizes para o Ensino Médico de 2001 no sentido de ampliar e qualificar o ensino fora do cenário e da lógica hospitalocêntrica e centrada na doença. ${ }^{2}$ Este objetivo de aprendizagem da disciplina é desenvolvido a partir da reflexão e aplicação do conceito ampliado de saúde e da noção de integração das intervenções de promoção, prevenção, curativa e de reabilitação.

Este deslocamento conceitual e metodológico do ensino objetiva desenvolver nos graduandos as competências de escuta e interpretação de diferentes percepções (individuais e coletivas) sobre o processo saúde-adoecimento e suas implicações na construção das necessidades e demandas por atenção à saúde. ${ }^{2}$ Portanto, formar médicos capazes de terem uma visão ampliada sobre saúde e doença e de superarem falsas dicotomias existentes no ensino e nas práticas em saúde entre o olhar sobre o coletivo e o individual e entre ações de promoção e atenção à saúde

$\mathrm{Na}$ contextualização e reflexão destes objetivos de aprendizagem este ensaio discutirá, inicialmente, a relação entre a dimensão coletiva e individual e, em seguida, traz uma reflexão sobre o ensino médico e as intervenções comunitárias. Esta discussão toma como referencia a importância de que o ensino médico se comprometa com a construção da cidadania e da autonomia dos indivíduos e coletividades sobre a saúde, o corpo e as ações de promoção e cuidado.

\section{Abordagem comunitária e a falsa dicotomia entre a dimensão coletiva e individual e entre intervenções de promoção e atenção à saúde.}

A análise da abordagem comunitária faz parte da trajetória de construção da promoção da saúde no campo da saúde pública e envolve uma complexa e imbricada relação entre o desenvolvimento da epidemiologia 
e a clínica. Caminhos que partem desde concepções preventivistas até visões mais atuais de promoção da saúde em que o conceito de empowermentse estabelece como uma imagem objetiva de um conceito ampliado das ações em saúde promovidas por sistemas de saúde.

Castellanos ${ }^{3}$ aponta a existência de uma tensão entre "o enfoque populacional versus enfoque individual" na estruturação da Epidemiologia como saber e prática. Esta dualidade se traduziria na definição e separação abstratas dos diferentes papéis das intervenções em saúde: “intervenções de caráter preventivo e de promoção sobre coletividades versus intervenções preventivas e, sobretudo, curativas aos indivíduos".

Em função dessa dualidade, as intervenções com enfoque populacional teriam um caráter mais intersetorial e relacionado aos projetos de desenvolvimento econômico e social. Já no caso das intervenções individuais, o objeto de trabalho fica reduzido a um conjunto de doenças e diagnósticos, agrupados segundo certa taxonomia, logo, perpetuando uma noção fragmentada e biológica do processo saúde-doença. Assim, o conhecimento e a prática na medicina não se articulam para constituir uma totalidade que envolva as relações históricas recíprocas e determinadas pela interação entre o homem e a natureza. ${ }^{3}$

Para Foucault ${ }^{4}$ há uma origem comum da medicina assistencial e da saúde pública. A relação estruturada entre ambas ocorre como duas faces distintas e distantes - a medicina individual e coletiva - de uma só origem eque se conformariam no que Foucault denominou de medicina social. A medicina social assumiria, portanto, o caráter de uma técnica de controle biológico do corpo, projetando este controle também sobre a vida social e o meio ambiente. ${ }^{4}$

Também entendendo este processo como fruto de uma matriz conceitual única, Rodriques ${ }^{5}$ sinalizou que a entrada em cena, a partir do final do século XVIII, do hospital - como locus da estruturação do saber anátomo-clínico - influenciou o desenvolvimento da epidemiologia e, consequentemente, da saúde pública. "Com efeito, no mesmo momento em que se inventa o hospital como instrumento terapêutico, são também estabelecidas as bases iniciais da atual legislação sanitária. [...] Submete-se o povo às normas higiênicas oficiais. Afinal, trata-se da preservação e da promoção da saúde. Neste caso, todos devem colaborar, aceitando as normas que advêm do profundo saber dos médicos." ${ }^{5}$

A convivência dos saberes anátomo-clínico e epidemiológico, e de suas práticas correspondentes, caracterizou-se pela complementaridade de funções, referentes, sobretudo, a essa manipulação explícita da vida social baseada nas relações com a saúde e a doença ${ }^{6}$

Além disso, esta inter-relação entre as noções de saúde e doença com o social submete-se a modificações de tempos em tempos, sempre que crises políticas recolocam a questão da qualidade de vida em cena e que são definidas diferentes concepções sobre a sociedade. ${ }^{6}$

Alguns modelos de intervenção em saúde desenvolvidas no século XX estabeleceram, objetivamente, a relação entre o coletivo e o individual e vice-versa. Entre os mais emblemáticos na saúde pública no século XX está o modelo de história natural da doença de Leavell $\&$ Clark $^{7}$ que inspirou, na década de 50 , o preventivismo. Sobre esta mesma égide destaca-se o conceito de "campo de saúde" apresentado no Relatório Lalonde de 1974 que introduziu o estilo de vida e os serviços de saúde no modelo de determinação das doenças. Este modelos ilustram certas tentativas de aproximação do social à saúde. ${ }^{8}$

Segundo o modelo da História Natural da Doença, a intervenção inicial no período pré-patogênico - a promoção da saúde - deveria se estruturar sobre o ambiente, as condições de vida e o comportamento pessoal, compreendendo a educação sanitária, a boa nutrição, o desenvolvimento da personalidade, as condições adequadas de moradia e recreação, e as condições de trabalho. Contudo, o meio ambiente desaparece subitamente às primeiras manifestações da doença, como se a alimentação, a habitação e outros fatores não interferissem na própria evolução e no curso do processo mórbido.

Rodrigues ${ }^{5}$ observa que "o homem representado no Modelo de História Natural, definitivamente, perdeu sua historicidade, tratando-se, portanto, de uma espécie de caricatura ingênua e muda, a exemplo de uma estátua". ${ }^{5}$

Kickbusch ${ }^{9}$ localizou, nas décadas de 60 e 70, o fortalecimento da epidemiologia do comportamento, na qual o Informe Lalonde foi um marco político e ideológico. $\mathrm{O}$ foco deste relatório eram os fatores de risco que, reduzidos ao nível individual, trouxeram a perspectiva de culpabilização das pessoas pelo seu adoecer como consequência negativa.

De modo pragmático, o documento de Lalonde buscava relativizar, e mesmo diminuir, o papel dos serviços de saúde em relação às ações sobre os outros elementos do campo da saúde. Esta hierarquia foi justificada ressaltando a melhor relação custo-efetividade das intervenções sobre os estilos de vida e sobre o meio ambiente quando comparada à atenção curativa pelos 


\section{Relato exper}

serviços de saúde.

Mais do que representar um marco teórico para o campo da Saúde Pública, o Relatório Lalonde pode ser reconhecido como um marco político-institucional da "Promoção da Saúde", ao vinculá-la diretamente ao processo de organização das políticas públicas.

A partir da década de 70, a chamada Epidemiologia Social, além de fazer questionamentos a estes modelos teóricos baseados nas redes de causalidade, formulava uma crítica direta à epidemiologia dos fatores de risco. Objetivava incluir os fatores socioeconômicos como responsáveis pelas transformações nos indicadores de morbimortalidade, ressaltando o papel de questões como as desigualdades sociais e econômicas e a pobreza em relação à saúde. ${ }^{8}$

A necessidade de intervenção no social foi acompanhada por uma sistematização e separação das estratégias de ação no setor sanitário como as propostas por Terris. ${ }^{3}$ Tratava-se de uma separação artificial das intervenções sanitárias que reforçam uma anteposição entre a atenção ao indivíduo e ao coletivo, pois fortalece a perspectiva coletiva como uma dimensão externa à dimensão individual ou vice-versa e, consequentemente, pode contribuir para o reforço de uma visão mecanicista e fragmentadora do processo saúde-doença.

\section{Perspectivas de integralidade na relação entre o coletivo e o individual nas ações de saúde}

Este desenvolvimento das relações entre o individual e o coletivo não avançavam na proposição de um modelo de atenção que situasse as práticas em saúde de forma que a historicidade do adoecer, na sua dimensão social e cultural, fosse também referência para o cuidado às pessoas nos seus processos de sofrimento individual e coletivo.

De certo modo, a inexistência de uma perspectiva integradora das intervenções em saúde diminui a legitimidade da própria ação de promoção ou de prevenção, visto que ela perde em conteúdos vivenciais, culturais e em representações do adoecer e do morrer, existentes nas sociedades.

Como, então, relacionar as dimensões individuais e o processo de adoecimento vivenciado pelas pessoas com os processos de desenvolvimento social e cultural dos grupos populacionais?

Neste sentido, Canguilhein ${ }^{10}$ ressalta a influência dos eventos sociais no processo saúde-doença-cuidado, chamando atenção para a necessidade de se considerar o exercício individual de produção de normatividade.

Este autor considerou que a saúde não se reduziria a um discurso normativo e a promoção da saúde não poderia estar restrita, apenas, a ordenar uma série de ações que geram bem-estar ou evitam riscos. É também dar condições de escolha e criação aos indivíduos. Logo, saúde não seria adaptação a normas, mas a capacidade de criar novas normas. ${ }^{10}$

Castiel11 contrapõe-se à visão de corpo-máquina e da saúde como evitação de riscos e a consequente normatização da saúde sobre a vida. Observa que esta discussão, minimamente, deveria tomar como base a evidência de que a saúde se configura como uma "macrocategoria multiforme", ao admitir que saúde se transmutaria de acordo com os contextos em múltiplas definições, com distintas repercussões e decorrências: "Especialmente, no âmbito humano, 'saúde', certamente, é diferente de 'vida'. Seria um pré-requisito para o viver, que inclui o prazer, a dor, a invenção, a criatividade, os arrebatamentos e isto, infelizmente não ocorre sem riscos, especialmente para a saúde". ${ }^{11}$

No discurso das organizações internacionais como a OMS e OPS - em sua pretensão de propor soluções generalizantes para diferentes países - a "Promoção da Saúde" foi assumida como uma abordagem afirmativa da saúde, em nítida oposição à noção de saúde como ausência de doença. ${ }^{12}$

Apesar deste discurso, que amplia o conceito de saúde e está voltado ao cotidiano, Castiel ${ }^{13}$ considera que persiste subjacente, na dinâmica de construção e operacionalização das noções sobre a "Promoção da Saúde", a concepção do "corpo-máquina", que se expressa na preocupação exacerbada com a procrastinação da morte e dos sinais de envelhecimento que o mundo ocidental persegue na atualidade.

Um processo de busca incessante por uma saúde perfeita que, segundo Sayd, ${ }^{14}$ vem ao encontro da expansão contínua da medicalização na nossa sociedade representa um tipo de subordinação das políticas e dos projetos sociais, voltados a uma melhor qualidade de vida da população, aos parâmetros epidemiológicos e aos princípios médico-sanitários. Deste modo, corre-se o risco da medicalização (e talvez de manipulação) da dinâmica social.É no interior deste campo que prospera o destaque dado aos comportamentos ligados ao estilo de vida, eventualmente conducentes a comportamentos de risco que devem ser evitados e a perda de valor do saber comum, popular e comunitário na construção da vida e da cidadania.

A Promoção da Saúde, apesar de estar direcionada 
à integração entre o individual e o coletivo, o meio ambiente e as condições de vida, os recursos necessários à sobrevivência e os hábitos e comportamentos, sua retórica desvincula estes aspectos de um processo histórico, ou melhor, de uma luta político-ideológica da sociedade na construção da cidadania e transfere grande parte desta responsabilidade política e social do Estado para determinados atores e ações setoriais (principalmente a saúde e a medicina).

A perspectiva de integrar dimensões de ordens distintas relacionadas à saúde humana torna-se intrinsecamente limitada se os instrumentais utilizados neste nível de intervenção permanecem restritos a um saber analítico, mecanicista e universalizante como se caracteriza o modelo biomédico. ${ }^{15}$ Nesse modelo, persiste a dicotomia entre problemas da mente e do corpo, concebidos sob uma determinada racionalidade científica que, por si só, restringe uma compreensão mais holística e complexa do que significa o sofrer do homem, limitando-se à observação de lesões estruturais ou funcionais em um corpo físico ou no comportamento social.

\section{A experiência do DMIF na aprendizagem do diagnóstico e intervenção comunitária como um construto da integralidade}

Como desdobramento desta visão das ações em saúde trabalha-se a ideia do desenvolvimento de uma nova e ampliada perspectiva semiológica e propedêutica da prática médica a partir da experiência de ensino-aprendizagem desenvolvida no DMIF-FCM nos últimos 14 anos. Reflete-se, portanto, sobre o aprendizado na comunidade como uma oportunidade de reflexão crítica das práticas em saúde, de ampliação do olhar e da escuta médica e de fomento da responsabilidade social dos profissionais.

Para integrar as diferentes dimensões e intervenções em saúde na aprendizagem da abordagem comunitária, o Departamento de Medicina Integral e Familiar (DMIF) compreende que mais do que deslocar o ensino para a comunidade é necessário incorporar uma pedagogia que leve os alunos a problematizar e refletir. Importa, por fim, desenvolver um saber e uma prática médicas que valorize o saber e as concepções sobre saúde e adoecimento construídos pelos profissionais, pessoas e comunidades.

A literatura da reforma do ensino médico indica que um currículo orientado pelos problemas da co- munidade está associado à significativa melhora de indicadores relativos à prevenção e a continuidade do cuidado sem prejuízo para a capacidade de diagnóstico de doenças e agravos na atuação de profissionais depois de formados. ${ }^{16}$

Ter a comunidade como um ambiente de aprendizagem implica que estudantes, professores, membros da comunidade e representantes de outros setores estejam envolvidos ativamente em toda a experiência educacional, isto é, na oferta de uma educação médica que seja relevante para as necessidades da comunidade. ${ }^{17}$

Para Troncon ${ }^{2}$ o maior engajamento da escola médica em atividades extramuros integra um novo paradigma educacional, fortemente centrado no estudante, que tem maior liberdade para definir seus objetivos instrucionais e, principalmente, para selecionar os recursos que deve utilizar para compor o seu aprendizado.

Este novo paradigma compreende a clara definição das competências, habilidades e atitudes a serem desenvolvidas e processos avaliativos condizentes com estes objetivos e uma rede de atenção à saúde orientada por uma APS qualificada. Esta qualificação da atenção primária constitui-se em uma base técnica, política e operacional essencial para expor os alunos a boas práticas no âmbito do SUS e da APS.

Contudo, o outro lado deste deslocamento do ensino para os cenários e contextos da APS é a responsabilidade da escola médica com os serviços de saúde, seus profissionais e com as necessidades de saúde dos moradores das comunidades atendidas. Sem esta via de mão dupla o processo de aprendizagem reproduz o caráter utilitarista e fragmentado do ensino tradicional realizado no ambiente hospitalar.

No caso da disciplina de Medicina Integral I, esta base técnico-política foi viabilizada com a expansão e qualificação da APS no Município do Rio de Janeiro ocorrida a partir de 2010 e pela atuação e responsabilização dos residentes e preceptores do Programa de Residência em Medicina de Família e Comunidade (PRMFC-UERJ) por equipes de saúde da família na rede municipal da Secretaria Municipal de Saúde do Rio de Janeiro. A inserção do PRMFC nas unidades implantadas e o desenvolvimento da preceptoria além de induzir a qualificação da rede de atenção também cria condições para uma melhor aprendizagem dos graduandos.

Desde sua introdução na grade curricular, a disciplina de Medicina Integral I objetiva o desenvolvimento de competências na abordagem comunitária e educação e promoção da saúde utilizando metodológicas ativas de aprendizagem. Sua base pedagógica é a pro- 
blematização, baseada no Método do Arco de Charlez Maguerez, de situações vividas pelos alunos.

São problematizadas, inicialmente, entrevistas com usuários ambulatoriais do Hospital Universitário Pedro Ernesto sobre suas compreensões e percepções do processo saúde e adoecimento. A partir destas narrativas e da vivência dos alunos, é construída uma visão polissêmica e ampliada do conceito de saúde. Com o desdobramento desta concepção de saúde são problematizados os conceitos de resiliência e de vulnerabilidade individuais, coletivas e programáticas.

Em um segundo momento, o desenvolvimento de competências relativas ao diagnóstico territorial e comunitário é feito por meio da vivencia dos alunos em diferentes territórios abrangidos pelas equipes de saúde da família (todos incluídos no PRMFC-UERJ). Os alunos, com a participação dos agentes comunitários de saúde, aplicam questionários adaptados por eles da Estimativa Rápida Participativa. O resultado das entrevistas são cruzados e analisados com base nos dados locais e regionais de sistemas de informação da Secretaria Municipal de Saúde do Rio de Janeiro, do Instituto de Planejamento do Município do Rio de Janeiro e do Instituto Brasileiro de Geografia e Estatística.

Estabelece-se um processo de aprendizado em espiral no qual os alunos são apresentados à Estimativa Rápida e Participativa(ERP) para elaboração do diagnóstico situacional do território. Este processo de análise e síntese culmina com a construção e discussão da árvore de problemas envolvendo profissionais, alunos e docentes. Dele decorre o planejamento participativo na construção e aplicação de ações de educação e promoção à saúde para os moradores do território.

A construção e o desenvolvimento desta ação educativa se baseiam em premissas como a importância de integrar os saberes técnico e popular, a valorização da escuta dos usuários e profissionais nas intervenções em saúde, a promoção da autonomia e resiliência e, do ponto de vista operacional, a preocupação com que uma atividade pontual da disciplina se integre com as ações longitudinais de abordagem comunitária que são planejadas e desenvolvidas pelas equipes no território.

Este processo tem a capacidade de ampliar a escuta e integrar o saber popular com o saber técnico-científico e os dados demográficos e epidemiológicos com as percepções dos moradores e dos profissionais para compreenderem e operarem criticamente sobre os determinantes de saúde e adoecimento no território.

Esta dinâmica de aprendizagem contrapõe-se a uma perspectiva monológica e normativa das intervenções em saúde e, particularmente, busca sobrepor-se a uma perspectiva de promoção da saúde que descontextualiza e culpabiliza as pessoas e comunidades em relação ao desenvolvimento de riscos e agravos.

Busca-se, portanto, enfatizar que as intervenções no campo da saúde, sejam elas de promoção, prevenção ou curativas, deve ter como objetivo promover espaços e condições de escolha e criação aos indivíduos implicados como sujeitos e protagonistas na construção da saúde. Logo, afirmar cotidianamente que saúde não seria adaptação a normas, mas a capacidade de criar novas normas. ${ }^{10}$

O departamento busca, deste modo, fazer com que a disciplina e a participação dos alunos representem uma contínua e progressiva integração das atividades acadêmicas com o processo de trabalho das equipes. Esta integração não se restringe a estas atividades disciplinares. Outras ações do DMIF contribuem neste sentido, tais como: a realização de oficinas de planejamento com as unidades de saúde, apoio matricial dos docentes às unidades, integração dos preceptores locais do PRMFC com a graduação, participação nos conselhos distritais de saúde e aproximação com a gestão municipal. Estas ações têm contribuído para o diálogo, trocas de informações e avaliações no desenvolvimento da integração ensino e serviço.

Esta tem sido uma trajetória longa e complexa, porém essencial para que os alunos compreendam a importância da escuta de indivíduos e da coletividade, da empatia, do diálogo, percebam a diversidade de concepções sobre o processo saúde-adoecimento e de seus determinantes e assim, assumam a responsabilidade social e profissional que envolve o médico como trabalhador em uma equipe. Além da importância para a aprendizagem do estudante, este percurso de diálogo e trocas contribui para o respeito, o empoderamento e a qualificação das equipes em relação aos saberes desenvolvidos no cotidiano do trabalho.

A aprendizagem da abordagem comunitária neste contexto técnico-político e operacional direciona as atividades para que os alunos construam, junto com as equipes, formas de conhecer a realidade de saúde e adoecimento das comunidades e, a partir desta compreensão, serem realizados o planejamento das ações de promoção e educação à saúde.

É a partir destas reflexões e ações que a disciplina de abordagem comunitária vem se desenvolvendo desde 2006, como a aprendizagem de um novo olhar, um novo estilo de pensamento nas práticas em saúde individuais e coletivas. Por que não dizer, uma nova 
semiologia e propedêutica do processo saúde e adoecimento, capaz de contextualizá-las como elementos de uma realidade individual e coletiva complexa, fruto de uma construção social, cultural e intersubjetiva.

\section{Referências}

1. Brasil. Ministério da Educação, Conselho Nacional de Educação, Câmara de Educação Superior. Diretrizes Curriculares Nacionais do Curso de Graduação em Medicina. Resolução CNE/CES n ${ }^{\circ} 4$, de 7 de novembro de 2001.

2. Troncon LEA. Ensino clínico na comunidade. Medicina, Ribeirão Preto, 32: 335-344, jul./set. 1999

3. Castellanos PL. Epidemiologia, Saúde Pública, Situação de Saúde e Condições de Vida. Considerações Conceituais. In: BARATA, RB, editor. Condições de vida e situação de Saúde. Rio de Janeiro: ABRASCO, 1997. p. 31-76.

4. Foucalt M. Vigiar e Punir: Nascimento da prisão. Petrópolis: Ed. Vozes, 1977, $280 \mathrm{p}$.

5. Rodrigues RD. A Crise da Medicina: Prática e Saber [dissertação]. Rio de Janeiro: Instituto de Medicina Social, Universidade do Estado do Rio de Janeiro, 1980.

6. Gonçalves RBM. Tecnologia e Organização Social das Práticas de Saúde: Características Tecnológicas de Processo de Trabalho na Rede Estadual de Centros de Saúde de São Paulo. São Paulo: Hucitec/Abrasco, 1994.

7. Leavell HR, Clark EG. Medicina preventiva. Rio de Janeiro: McGraw-Hill do Brasil, 1976.
8. Paim JS. Abordagens Teórico-Conceituais em Estudos de Condições de Vida e Saúde: Notas para Reflexão e Ação. In: BARATA, RB, editora. Condições de Vida e Situação de Saúde. Rio de Janeiro: ABRASCO, 1997. p. 7-30.

9. Kickbusch I. Promoción de la Salud: Una Perspectiva Mundial. In: Organización Panamericana de la Salud, editor. Promoción de la Salud: una antologia. Washington, DC: OPS, 1996, p. 15-24

10. Canguilhen G. O Normal e O Patológico. 2. edição. Rio de Janeiro: Ed. Forense Universitária, 1982.

11. Castiel LD. A medida do possível... saúde, risco e tecnobiociências. Rio de Janeiro: Contra Capa Liv. Ed. FIOCRUZ, 1999.

12. Testa M. De llusiones Perdidas y Esperanzas Futuras. Saúde em Debate, 2000, v. 24, n. 55, p 5-31.

13. Castiel LD. Dédalo dentro do dédalo? Identidade cultural, subjetividade e promoção de saúde. 50 Congresso Brasileiro de Saúde Coletiva; 2000, Salvador: Anais... Nordeste Digital, 2000.

14. Sayd JD. Novos Paradigmas e Saúde. - Notas de Leitura. Physis: Revista Saúde Coletiva, 1999, v. 9, n. 1, p. 113-121.

15. Camargo Jr. KR. A Biomedicina. Physis: Revista Saúde Coletiva, 1997, v.7, n.1, p. 45-68.

16. Mennin S, Mennin RP. Community-based medical education. The Clinical Teacher. Oxford (UK), Blackwell Publishing Ltd 2006. 2006; 3: 90-96.

17. Tamblyn R, Abrahamowicz M, Dauphinee D, et al. Effect of a community oriented problem based learning curriculum on quality of primary care delivered by graduates: historical cohort comparison study. BMJ Online First bmj.com, October 2005

18. Silveira CH. Notas sobre a metodologia da Estimativa Rápida. Genebra: Organização Mundial de Saúde, 1998. 